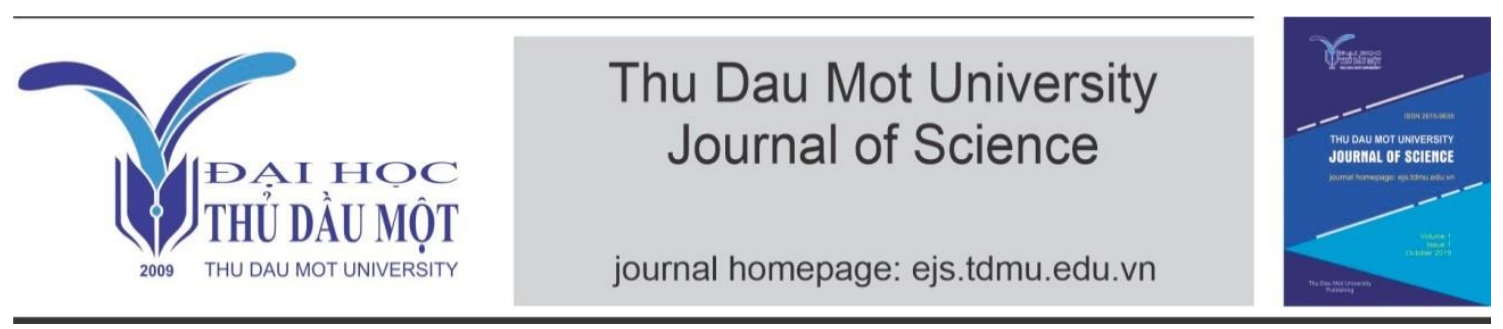

\title{
A review on bioethanol production using lignocellulosic biomass
}

\author{
by Truơng Nguyễn Phuơng Vi (Thu Dau Mot University)
}

\author{
Article Info: $\quad$ Received 31 Mar. 2021, Accepted 2 June 2021, Available online 15 June 2021 \\ Corresponding author: vitnp@tdmu.edu.vn \\ https://doi.org/10.37550/tdmu.EJS/2021.02.201
}

\begin{abstract}
Declining supplies of fossil fuels, increasing population, global industrialization, and demand for transportation fuels have triggered an increase in the demand for renewable energy sources. To address such problems most of the green research in recent years has focused on the development of bioethanol (23 MJ/L) as a substitute to conventional gasoline (34.3 MJ/L) based fuels owing to the similarity in energy density values in addition to several other advantages (American Council on renewable energy, 2010). Second-generation biofuels are derived from lignocellulosic biomass or woody crops, mostly coming from agricultural residues. Extraction of fuel from such biomass is difficult because of their recalcitrant nature (corn stover, rice straw, wheat straw, sugar cane, and sweet sorghum). Lignocellulosic fuel has the potential to solve several problems (food competing with fuel) that are currently associated with first-generation biofuels. Moreover, lignocellulosic fuels can supply a larger proportion of the global fuel leading to sustainability at a lower cost, and with greater environmental benefits (Liz Marsall, 2009). The production of ethanol from the complex sugars in leaves and stalks is a promising strategy to radically broaden the range of possible ethanol feedstock.
\end{abstract}

Keywords: lignocellulose, bioethanol, biomass, pretreatment, hydrolysis, fermentation

\section{Introduction}


Lignocellulosic biomass feedstock typically contains $55-75 \%$ by dry weight carbohydrate, that are polymers of five- and six-carbon sugar units. Most of all these carbohydrates can be converted to ethanol via biotechnology. As many as possible, these carbohydrates need to be converted to maximize ethanol production. Besides, the complex structure of lignocellulose necessitates the pretreatment of biomass before fermentation.

However, the problem with lignocellulosic feedstock is that it has useful sugars locked in by lignin, hemicellulose, and cellulose. Their accessibility is further hindered by the presence of lignin in the matrix. These are complex carbohydrates and the considerable variation in the relative amounts of each of the structural components, cellulose, hemicellulose, and lignin in the cell walls as well as physical properties such as cellwall thickness and porosity would depend on the specific plant species. Previous research has reported that plant cell walls in lignocellulosic biomass are highly resistant to chemical, physical, and enzymatic degradations (Charlier et al., 2012). Therefore, pretreatment is required for disrupting the recalcitrant structure of the plant cell walls allowing easy access for sugars that later can be fermented to produce ethanol in the same way as that of the first-generation biofuels.

There are several technologies available for the conversion of lignocellulosic material to ethanol. Simultaneous saccharification and fermentation (SSF) were studied for cellulose conversion to ethanol. By combining hydrolysis and fermentation in the same reactor, glucose is rapidly removed before it can inhibit further hydrolysis (Kerstin Hoyer, 2013). SSF has been a successful method in the production of lignocellulosic ethanol. Another advantage is the reduction in material cost since only one reactor is needed. However, the drawback with SSF is the cooperation between the optimal conditions for hydrolysis and fermentation. A common temperature for $\mathrm{SSF}$ is $37^{\circ} \mathrm{C}$, which is not optimal for either the cellulases or the fermenting microorganism. A second drawback is enzyme and microbe inhibition caused by the produced ethanol. This inhibition can be a limitation to achieve higher ethanol concentrations (Kim Olofsson, 2008).

To achieve cost-effective conversion of biomass to ethanol, the microorganism used to ferment the monomeric sugars must give a high ethanol yield and productivity. The yeast Saccharomyces cerevisiae has been widely used for ethanol fermentation for centuries (Kerstin Hoyer, 2013). Under anaerobic conditions, it ferments glucose to ethanol and is known for its superior tolerance to ethanol (Kerstin Hoyer, 2013). The main disadvantage of $S$. cerevisiae is its narrow substrate utilization range. S. cerevisiae only ferments certain hexose sugars, but cannot utilize the pentose sugars in the substrate efficiently as it lacks both a xylose-assimilation pathway and adequate levels of key pentose phosphate pathway (PPP) enzymes (Wyman et al., 1986, Micky Anak Vincent, 2010; Kerstin Hoyer, 2013). An attempt was made to surmount the 
aforementioned challenges in the lignocellulosic ethanol production via developing an effective pretreatment methodology for improving the efficiency of the process.

\section{Lignocellulosic biomass}

\subsection{Plant cell wall structure and compositions}

Lignocellulose is the main component of plant cell walls and provides a structural and protective barrier for the plant cells (Figure 1.1). Lignocellulosic material is the threedimensional polymeric material, which is composed of many components, such as carbohydrates (cellulose and hemicellulose), lignin, extractives, and others in minor quantities. Carbohydrates and lignin make up a major portion of lignocellulosic biomass. Carbohydrates are composed of polymers of five- and six-carbon sugars, which typically account for $55-75 \%$ of biomass weight. Lignin is a source of polyphenolic compounds. Carbohydrate and lignin contents can be determined by a two-stage acid hydrolysis method developed by NREL (Sluiter et al., 2008).

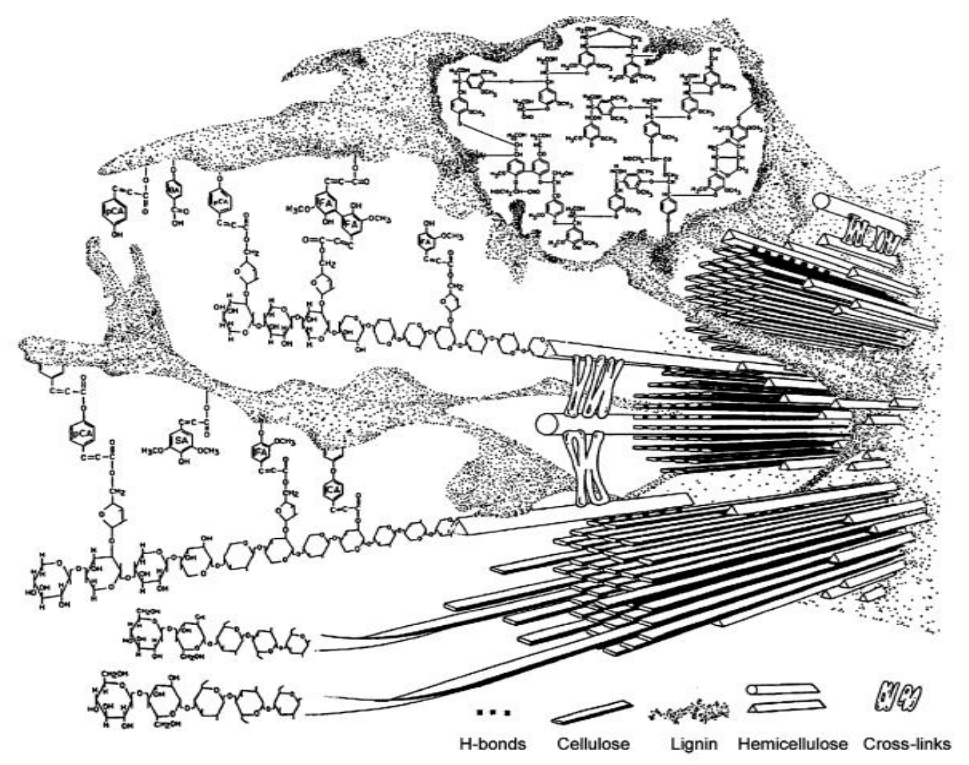

Figure 1. Schematic representation of plant cell wall showing linear cellulose and branched hemicelluloses chains surrounded by a lignin matrix (Martinez et al., 2009).

\section{Cellulose}

Cellulose provides strength and flexibility to the plant cell wall and the fibers. Cellulose is the main constituent of lignocellulosic biomass. The cellulose content of lignocellulosic biomass varies between species in the range of $30-0.5$ (on a dry wt. basis). Cellulose is a linear polymer of $\beta$-D-glucopyranose units (Fig. 1). These glucose units are linked together by $\beta-1,4-$ glycosidic linkages. Strictly speaking, the cellobiose unit is the repeating unit of the cellulose chain, with a length of $1.03 \mathrm{~nm}$ (Fengel and 
Wegener, 1984). The degree of polymerization (DP) of native cellulose is in the range of 500-15000.

Cellulose in the cell wall forms long, oriented microfibrils, which may coalesce into larger and longer fibrils (Brain H. Davison et al., 2013). The cellulose microfibrils are hydrophobic and can be highly crystalline, features that contribute greatly to the recalcitrance of biomass (Brain H. Davison et al., 2013). Bundles of molecules of cellulose are aggregated together in the form of microfibrils, in which highly ordered (crystalline) regions alternate with less ordered (amorphous) regions (Fig. 1). Microfibrils build up fibrils and cellulose fibrils (Sjostrom, 1993). Cellulose molecules are completely linear and have a strong tendency to form intra- and intermolecular hydrogen bonds (Sjostrom, 1993). Figure 1 illustrates intra- and inter-molecular hydrogen bonds between glucose units and cellulose molecules. It has been suggested that various hydrogen bonds may be responsible for the formation of the crystalline structure of cellulose. Each cellulose chain approximates to a flat ribbon, with alternate glucose units facing in opposite directions. They are locked in this position by a hydrogen bond between a hydroxyl group $\left(\mathrm{O}_{3}-\mathrm{H}\right)$ of one glucose unit and the ring oxygen $\left(\mathrm{O}_{5}\right)$ of the next. All the cellulose molecules lay parallel, hydrogen-bonded edge to edge (Javis, 2003).

\section{Hemicellulose}

The structure of hemicellulose consists of heterogeneous polysaccharides formed through biosynthesis. They are a class of polysaccharides with variable composition and structure depending on the plant source (Brain H. Davison et al., 2013). For example, hemicellulose isolated from herbaceous grass species, such as switchgrass, comprise glucuronoarabinoxylans which are complex, branched polysaccharides composed mainly of pentose (five carbon) sugars. On the other hand, hemicelluloses in softwood species, such as pines, are predominantly composed of galactose glucomannans, which have a backbone of D-glucopyranose and $\beta-1,4$ linked D-mannopyranose units. Similar to cellulose, hemicellulose is one of the supporting materials in the cell walls. They consist of five different major sugar units, including D-glucose, D-xylose, D-galactose, L-arabinose, and D-mannose plus small amounts of L-rhamnose, D-glucuronic acid, 4O-methyl-D-glucuronic acid, and D-galacturonic acid. Among these sugars, D-xylose (a pentose or five-carbon sugar) is the major component of hemicellulose in a wide variety of lignocellulosic biomass species. Hemicellulose has a DP of only about 200, which is much lower than that of cellulose. In order to achieve high lignocellulosic biomass to ethanol process yield, hemicellulose utilization is necessary since the contribution of hemicellulose is $40-0.5$ of total carbohydrates in high biomass.

Hemicellulose is easier to be removed from the cell-wall matrix than the other components during pretreatment under acidic conditions. The downside of this ease of 
removal from the cell wall is that the hydrolyzed free sugars and oligosaccharides will continue to react under these pretreatment conditions and degrade into undesirable compounds for biological conversion. These include furans which, in addition to being a loss of sugars and the overall process yield, are also inhibitory to subsequent biological conversion processes. While most of the hemicellulose can be easily removed by pretreatment, the covalent cross-linkages appear to create a residue of hemicellulose or lignin carbohydrate complexes, which may continue to shield or partially protect cellulose microfibrils from attack. Hemicellulose can be substituted at hydroxyl groups with different side-chain chemical groups, primarily acetyl groups. The acetyl groups easily hydrolyze under thermal or acidic conditions to form dilute acetic acid, which lowers the $\mathrm{pH}$ of the pretreatment liquor and increases the rate of hydrolysis. Thus, even a hot water pretreatment has the benefit of actually being a mild auto catalyzed dilute acid pretreatment. The negative aspect is that the acetate formed must be neutralized before further processing steps such as enzymatic hydrolysis and also can be inhibitory to many fermentative microorganisms.

\section{Lignin}

Lignin is a polyphenolic compound, and its name was derived from the Latin word lignum, which means wood (Sarkeanen and Ludwig, 1971). Figure 2 presents the three lignin units: coniferyl, sinapyl, and p-coumaryl alcohol units. Lignin molecules are a high-molecular-weight natural product and highly linked to each other. One of its functions in plants is to hold cellulosic fibers together and to impart strength to the lignocellulosic bio-composite (Glasser, 1980).

The two main types of lignins are the guaiacyl lignins, in which alcohol precursors with one methoxy predominate (characteristic of softwood), the guaiacyl-syringyl lignins, in which both methoxyl- and dimethoxy-substituted alcohols are present. In general, plant materials can be classified into three types, which are hardwood, softwood, and grass species. Hardwood and grasses contain guaiacyl-syringyl lignins and also acids, such as p-hydroxybenzoic acid esterified to the lignin macromolecule. The terms softwood and hardwood are used to refer to the taxonomical division that separates a species. However, they have little to do with the actual hardness of the wood. Hardwood trees have broad leaves and deciduous; that is, they lose their leaves at the end of the growing season. Hardwoods are angiosperms, which are known as flowering plants. Softwood trees are conifer, with needles or scale-like foliage, and are not deciduous. Softwoods are gymnosperms, a group that encompasses most trees known as evergreens. Pretreatment of hardwood has several advantages like the relative ease of delignification, the presence of highly acetylated xylan that can accelerate autohydrolysis during pretreatment, and the low ash content (silica). Hardwood lignin 
has a lower DP compared to softwood lignin and higher methoxyl content. In contrast, softwoods lignin are considered to be difficult to be delignified (Sjostrom, 1993).

The utilization of lignin is an increasingly important field. The field of lignin utilization includes (1) lignin as fuel (due to its high energy content of 11,300 Btu/1b); (2) lignin as feedstock for polymeric products; (3) lignin as a source of high-value low-molecularweight phenolic compounds.

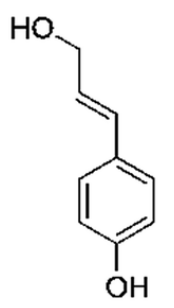

p-coumaryl

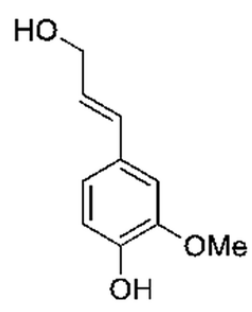

coniferyl

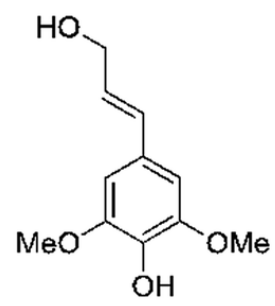

sinapyl alcohol

Figure 2. The three-building units of lignin

\section{Extractives}

A large variety of wood components that are soluble in neutral organic solvents or water are called extractives (Sjostrom, 1993). The extractives comprise an extraordinarily large number of individual compounds of both hydrophilic types and lipophilic. The content of extractives varies but usually is less than $10 \%$ of the dry weight of biomass. Quantitative determination of extractives is carried out by standardized methods such as extraction with organic solvents (hexane, dichloromethane, diethylether, acetone, or ethanol) and water. Extractives analysis typically consists of two steps, which determine water and ethanol extractives. Water-soluble materials may include nonstructural sugars, nitrogenous materials, inorganic materials. Ethanol-soluble materials include chlorophyll, waxes, or other minor components (Sluiter et al., 2008). Extractives are very important because they have high potential as a source for the production of valueadded products. Some extractive is toxics, which allows the wood to resist attack by fungi and termites.

\subsection{Bioethanol production using lignocellulosic biomass}

\section{Pretreatment}

Pretreatment is one of the most costly steps in the conversion of lignocellulose to sugars. Pretreatment often produces biological inhibitors, which impact the cost of fermenting the resulting sugars. As a result, much attention is directed at developing low-cost and effective pretreatment methods (Yogi Goswami, 2016). The goal of the pretreatment process is to increase the surface area of lignocellulosic material, making the polysaccharides more susceptible to hydrolysis. Therefore, size reduction is an integral part of pretreatments. 
Depending on the feedstock material, most pretreatment techniques require the starting substrate to be prepared using a mechanical size reduction step so that the substrate is sized appropriately for handling. The largest substrate particle size known to be appropriate for any pretreatment technique is that of commercial wood chips, or roughly $1-3 \mathrm{~cm}$ length and a width of 0.5 to $1 \mathrm{~cm}$ thickness.

The mechanisms by which pretreatment improves the digestibility/hydrolysis of lignocellulose are not well understood. Pretreatment effectiveness is correlated with the removal of hemicellulose and lignin. Lignin solubilization is beneficial for subsequent hydrolysis but also results in a by-product that inhibits enzyme activity. Some pretreatments are thought to decrease the crystallinity of cellulose, which improves activity, but this does not appear to be the key for many effective pretreatments. A large variety of pretreatment processes developed can be broadly classified as physical, chemical, biological, and/or a combination of the aforementioned pretreatments.

\section{Physical pretreatment}

Physical pretreatment is used for reducing the crystallinity of cellulose. It includes chipping, grinding, and/or milling. Milling effectively reduces the particle size and cellulose crystallinity due to the shear forces generated during milling. There are some different methods of milling such as two-roll milling, colloid milling, hammer milling, and vibratory 'milling which are used to improve the enzymatic hydrolysis of lignocellulosic biomass. The ball milling method is found to be more effective in reducing the particle size of biomass leading to an improvement in digestibility (Adepu Kiran Kumar and Shaishav Sharma, 2017). Physical pretreatment is usually occurred before the following processing step and desired particle size depending on these subsequent steps. For mechanical pretreatment, some factors such as capital cost, operating cost, scale-up possibilities, and depreciation of equipment are very important (Harmsen et al., 2010).

\section{Alkaline pretreatment}

Typical examples include diluting base, alkaline solvent, ammonia, $\mathrm{SO}_{2}, \mathrm{CO}_{2}$, other basic chemicals, and $\mathrm{pH}$-controlled hydrothermolysis. Several dilute-acid pretreatment techniques, including dilute mineral acids (sulfuric acid, nitric acid, hydrochloric acid, phosphoric acid, and peracetic acid) were also reported. A wide range of feedstock such as hard and softwood biomass, agriculture residues, and wastepaper was investigated. Of all acid-based pretreatment techniques, sulfuric acid has been most extensively studied, because of its inexpensive and effective nature.

Alkaline pretreatment has been studied for many years as important for improving the enzymatic digestibility of lignocellulosic materials. $\mathrm{NaOH}$, ethylenediamine, and $\mathrm{Ca}(\mathrm{OH})_{2}$ were used as pretreatment reagents. Alkaline pretreatment causes strong swelling, decreases the DP of cellulose, lowers the lignin content, and increases the 
surface area. Saponification of intermolecular ester bonds was also reported (Fan et al., 2011). It was observed that steeping various straws in $1.5 \% \mathrm{NaOH}$ for $24 \mathrm{~h}$ increased their ruminant digestibility from an initial 30-40 to 60-70\%. This process was patented by Beckmann and was used extensively in Europe during World Wars I and II. NaOH treatment causes a significant amount of hemicellulose solubilization (Sjostrom, 1981) which depends on the substrate and operating conditions.

Alkaline pretreatment is a delignification process. A significant amount of hemicellulose would be solubilized as well. The effectiveness of alkaline pretreatment appears to vary depending on substrate and treatment conditions. Overall, alkaline pretreatment is more effective on lignocellulosic material and herbaceous crops than on wood materials.

Ammonia was also used as a reagent for pretreatment, even though the enhancement of biomass hydrolysis obtained is less than that of the $\mathrm{NaOH}$ treatment in general. Nonetheless, as compared to $\mathrm{NaOH}, \mathrm{NH}_{3}$ has a number of desirable characteristics as a pretreatment reagent. $\mathrm{NH}_{3}$ is relatively cheap and is one of the widest chemicals used in the commodity market. It can be recycled easily due to its high volatility. In $\mathrm{NH}_{3}$ based pretreatment, theoretically, more than $99 \%$ of $\mathrm{NH}_{3}$ can be recovered and reused. The residual $\mathrm{NH}_{3}$ in the biomass can serve as a nitrogen source for the subsequent fermentation. Aqueous $\mathrm{NH}_{3}$ is known to be effective for delignification of lignocellulosic material, cleavage of the carbon-to-carbon (C-C) bonds in lignin, as well as the ether (C-O-C) and ester (C-O-O-C) bonds in the biomass. The enzymatic digestibility of lignocellulosic material is increased remarkably by ammonolysis as a result of breaking the linkage between lignin and hemicellulose and increasing the surface area (Kim et al., 2013).

Combined physical and alkaline pretreatment can increase the efficiencies of the pretreatment compared to alkaline pretreatment alone. However, the cost for operation and equipment should be considered when scaling up the process.

\section{Enzymatic hydrolysis}

Enzymatic hydrolysis was developed to better utilize both cellulose and hemicellulose from lignocellulosic biomass. Cellulose is a homopolysaccharide of glucose linked by B-1,4-glycosidic bonds. Therefore, enzymatic hydrolysis of cellulose proceeds in different steps to break glycosidic bonds by the action of a system of enzymes known as cellulase. Native cellulose is hydrolyzed by the isoenzymes cellobiohydrolase I and cellobiohydrolase II to yield cellodextrins and cellulose. The cellodextrins are further hydrolyzed to cellobioses, a disaccharide of glucose, by the isoenzymes endoglucanase I and endoglucanase II. The cellobiose is hydrolyzed to monosaccharides by $\beta$ glucosidase. The system of enzymes also usually contains hemicellulase to hydrolyze any hemicellulose not solubilized by pre-hydrolysis (Charlie et al., 1996). 
A variety of fungi and bacteria produce cellulase both aerobically and anaerobically. The aerobic mesophilic fungus Trichoderma reesei and its mutants have been the most intensely studied sources of cellulase. Other fungal cellulase producers include $T$. viride, T. lignorum, T. koningii, Penicillium spp., Fusarium spp., Aspergillus spp., Chrysosporium pannorum, and Sclerotium rolfsii. The problems with enzymatic hydrolysis include relatively low specific activity, low rates of conversion, and sensitivity to end-product inhibition. The low specific activity leads to high enzyme loading requirements: approximately $1 \mathrm{~kg}$ of enzyme for the hydrolysis of $50 \mathrm{~kg}$ of cellulose fibers. Conversion rates are as low as $20 \%$ in $24 \mathrm{~h}$. Up to seven days are required to digest lignocellulose. A technique described in the next section, the simultaneous hydrolysis of cellulose and fermentation of hexose as it is released, can substantially overcome substrate inhibition (sugar) inhibition.

\section{Fermentation}

Fermentation is a biological process in which enzymes produced by microorganisms catalyze energy-releasing reactions that break down complex organic substrates. Anaerobic conditions, in which oxygen is present, are also possible. Fermentation can produce a wide variety of chemicals, although most of them are organic acids and alcohols. Most microorganisms used in commercial fermentation require six-carbon sugars (hexose) or disaccharides as substrate, although the microbial world contains organisms that can break down virtually any organic compound. Recent technological advances have made fermentation of five-carbon sugars (pentose) possible. Several factors limit the use of fermentation technology in the production of chemicals. Production rates by microorganisms in aqueous media of low-solids volume are inherently low. The microorganisms are sensitive to both inhibitors and operating conditions, especially temperature and $\mathrm{pH}$. Most fermentation requires aseptic conditions, which can be difficult to achieve in large-scale operations.

A major emphasis in the fermentation industry is the production of ethanol, which is marketed to both fuel and drinkable industries. Ethanol fermentation yields for a variety of carbohydrate feedstock are summarized in Table 1. The maximum theoretical yield of ethanol is 0.51 (mass ethanol/mass carbohydrate, corresponding to $51 \%$ of the carbohydrate converted to ethanol on a mass basis) with the rest being $\mathrm{CO}_{2}$. Typically about 5-12 wt.\% of the carbohydrate is converted to microorganisms (yeast cells). Thus, practically not more than $47 \mathrm{wt} . \%$ of the fermented carbohydrate is converted to ethanol. Of course, other by-products (glycerol, 1, 3 propanediol) can also be produced during sugar fermentation.

TABLE 1. Ethanol yields from various biorenewable resources

\begin{tabular}{lcc}
\hline & Feedstock & Ethanol yield $(\mathrm{L} / \mathrm{t})$ \\
\hline Apples & & 64 \\
Barley & 330 \\
\hline
\end{tabular}




\begin{tabular}{lc}
\hline Cellulose & 259 \\
Corn & $355-370$ \\
Grapes & 63 \\
Jerusalem artichoke & 83 \\
Molasses & $280-288$ \\
Oats & 20.65 \\
Potatoes & 96 \\
Rice (rough) & 332 \\
Rye & 329 \\
Sorghum (sweet) & $44-86$ \\
Sugar beets & 88 \\
Sugar cane & $160-187$ \\
Sweet potatoes & $125-143$ \\
Wheat & 355 \\
\hline
\end{tabular}

Source: D. L. Klass, 1998, Biomass for Renewable Energy, Fuel, and Chemicals, Academic Press.

These technologies are based on microbial and enzymatic processes for producing sugars from biomass and are subsequently converted into alcohol and other solvents. Lignocellulosic biomass such as corn stover, rice straw, wheat straw holds enormous promise. Challenges in the conversion of biomass to bioethanol include reduction of production costs requiring improved cellulose and hemicellulose conversion to sugar, combined xylose and glucose fermentation, lower pretreatment energy requirements, and an efficient separation process for alcohol (Naik et al., 2009).

\section{Ethanol production from lignocellulose material using Saccharomyces cerevisiae D5A}

$S$. cerevisiae, as a model of eukaryotic cells, has been extensively studied in fundamental biological science. Its genome sequencing was completed in 1996 and about 6000 potential protein-coding genes were identified (Andrea A. Duina et al., 2014), which provides a unique tool for molecular microbiologists to better understand this species at its molecular level and develop modified strains with new metabolic pathways, such as the recombinants capable of metabolizing pentose and fermenting lignocellulosic hydrolysates (Ho et al., 1998). It also allows biochemical engineers to operate their fermentation plants more efficiently and economically by manipulating this biocatalyst at its process-control level and improve its product yield that is extremely important for those bulk fermentation products, ethanol as an instance.

The production of ethanol, a typical primary metabolite, is tightly associated with the growth of yeast cells. Therefore, many considerations involved in ethanol fermentation processes, including fermentation kinetics and process optimization, are based on the growth of yeast cells. S. cerevisiae propagates by budding, which is an asymmetric process and schematically illustrated in Figure 3 (Patnaik, 2003). The parent and daughter cells can be distinguished and show different behaviors during the reproduction cycle. It is known that the size of the buds at division, the newborn daughter cells, is smaller than their parents, and they need a longer time to be ready for 
budding than that their parents require.

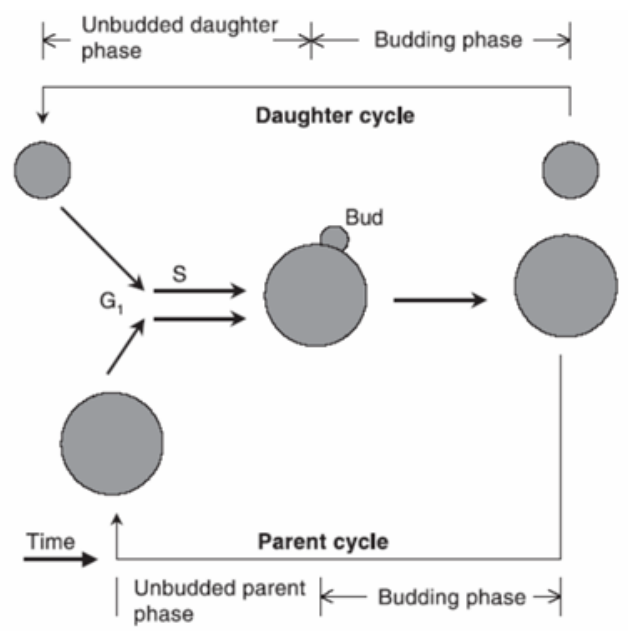

Figure 3. Diagram of the asymmetric budding cycle of S. cerevisiae (Patnaik, 2003)

Hartwell and Unger (1977) investigated the unequal division in S. cerevisiae and the control mechanism for the cell cycle. Later research further found that the cell cycling characteristics of $S$. cerevisiae can be smoothly explained by the concept of critical cell mass for the initiation of division (Bellgardt, 1994a, b). According to this concept, the generation time of the newly born daughter cells is longer than that of their parent cells, because they need to accumulate more cell mass. This specific growth pattern of S. cerevisiae was believed to be the intrinsic mechanism triggering the sustained oscillations of biomass, sugar, dissolved oxygen, and evolved $\mathrm{CO}_{2}$, and so on during the continuous aerobic cultures of $S$. cerevisiae.

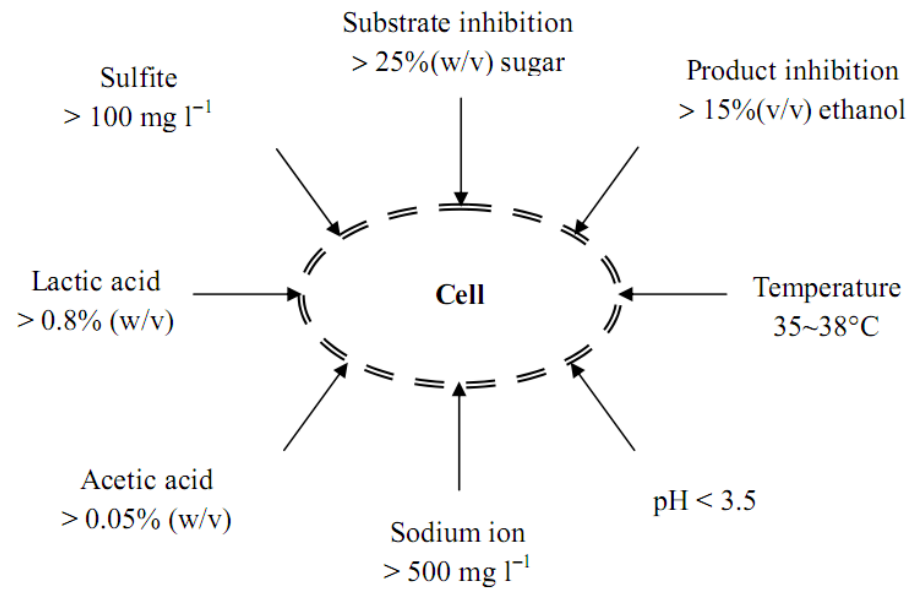

Figure 4. Environmental stresses exerted on S. cerevisiae during ethanol fermentation (Ingledew, 1999)

However, under anaerobic ethanol fermentation, yeast cells suffer from various stresses. Some of them are environmental, while the others are generated by the yeast cells themselves, for example, ethanol accumulation, and correspondingly strong inhibition 
on yeast cell growth and ethanol fermentation. The yeast cells in ethanol fermentations are under harsh conditions compared with their aerobic cultures in which the process parameters are optimized with optimum $\mathrm{pH}$, sufficient nutrients, and very low ethanol that does not exert inhibition. Their metabolic activities are seriously affected, especially under industrial conditions in which the optimum conditions for growth and fermentation are overwhelmed by pursuing economic benefits (Ingledew, 1999). Figure 4 illustrates some stresses that yeast cells could experience in ethanol fermentations. Many of them are synergistic, which will exacerbate their negative impacts on yeast cells simultaneously. Stresses, especially ethanol inhibition, and the lag response of yeast cells to it are assumed to be the main reason triggering the parameter oscillations during the continuous ethanol fermentation under a VHG condition.

Ethanol fermentation is a biological process in which organic material, carbohydrates, is converted by microorganisms to simpler compounds such as ethanol (Yan Lin and Shuzo Tanaka, 2005). S. cerevisiae produces ethanol in high yield with high ethanol tolerance (over $100 \mathrm{~g} / \mathrm{L}$ ) and has been reported for some strains and media. In addition, this organism has proven to be robust to other inhibitors; therefore, it is suitable for fermentation of lignocellulosic biomass (Kim Olofsson et al., 2008).

\section{Simultaneous Saccharification and Fermentation (SSF)}

The idea of performing simultaneous saccharification and fermentation was put forward by Gauss et al. in a patent from 1973. Until now, simultaneous saccharification and fermentation (SSF) is one of the process options for the production of ethanol from lignocellulosic materials. The SSF process combines saccharification and fermentation to overcome the end-product inhibition that occurs during the hydrolysis of cellobiose. By combining hydrolysis and fermentation in the same reactor, glucose is rapidly removed before it can inhibit further hydrolysis.

SSF has been a successful method in the production of lignocellulosic ethanol (Taherzadeh and Karimi, 2007). The major advantage with SSF is that the released glucose is immediately consumed by the ethanol-producing organism, thus avoiding cellulase inhibition (Galbe and Zacchi, 2002) and avoiding a potential loss of sugar (Kim Olofsson et al., 2008). Furthermore, the combination of hydrolysis and fermentation decreases the number of vessels needed and thereby investment cost (Kim Olofsson et al., 2008). Reports have shown that SSF results in higher ethanol yield with lower enzyme addition compared to SHF (Taherzadeh and Karimi, 2007).

However, the drawback with SSF is the compromise between the optimal conditions for hydrolysis and fermentation. A common temperature for SSF is $37{ }^{\circ} \mathrm{C}$, which is not optimal for either the cellulases or the fermenting microorganism. Furthermore, the yeast cannot be reused in SSF due to the problem of separating the yeast from the lignin 
after fermentation. The enzymes are equally difficult to be reused (Kim Olofsson et al., 2008). Recirculation of enzymes is equally difficult since the enzymes bind to the substrate, although partial desorption can be obtained after the addition of surfactants. (Kim Olofsson et al., 2008). Another drawback is enzyme and microbe inhibition caused by the produced ethanol. This inhibition can be a limitation to achieve higher ethanol concentrations (Taherzadeh and Karimi, 2007). The hydrolysis temperature, time, and acid concentration influence the generation of fermentation inhibitors.

\section{Conclusion}

Limited supplies of fossil resources, climate change due to carbon dioxide accumulation in the atmosphere, and increased demand for fuels and chemicals have triggered an increase in the utilization of diverse renewable feedstock. To implement the production of a wide range of fuels, chemicals, and materials from renewable sources, almost green research in recent years has focused on the development of renewable fuels and biobased chemicals as a substitute for conventional fossil fuels (gasoline and diesel) and petroleum-based chemicals. In particular, cellulosic fuel ethanol, a second-generation biofuel, has the potential to solve several problems, including limited feedstock availability and food competition with fuel, that are currently associated with firstgeneration biofuels such as fuel ethanol from corn starch or sugarcane. Cellulosic ethanol can be produced from inexpensive and abundant lignocellulosic materials such as woody biomass and herbaceous biomass. Therefore, it is currently believed that cellulosic ethanol can meet a larger proportion of global transportation fuel demand in the near future.

\section{References}

Adepu Kiran Kumar and Shaishav Sharma (2017). Recent updates on different methods of pretreatment of lignocellulosic feedstocks: a review. Bioresour. Bioprocess. (2017) 4:7 DOI 10.1186/s40643-017-0137-9.

Andrea A. Duina, Mary E. Miller and Jill B. Keeney (2014). Budding Yeast for Budding Geneticists: a Primer on the Saccharomycess cerevisiae Model System. Genetic. 197(1), 33 -48 .

Badger, P.C. Ethanol from cellulose: A general review. In Trends in New Crops and New Uses; Jnick, J.,

Whipkey, A., Eds. (2002). ASHS Press: Alexandria, VA, USA. pp. 17-21

Bellgardt KH (1994a). Analysis of synchronous growth of yeast. Part I: Development of a theoretical model for sustained oscillations. J Biotechnol, 35: 19-33.

Bellgardt KH (1994b). Analysis of synchronous growth of yeast. Part II: Comparison of model prediction and experimental data. J Biotechnol, 35: 35-49. 
Brian H. Davison, Jerry Parks, Mark F. Davis and Bryon S. Donohoe (2013). Plant cell walls: Basics of structure, chemistry, accessibility and the influence on conversion. Aqueous Pretreatment of Plant Biomass for Biological and Chemical Conversion to Fuels and Chemicals, First Edition. Edited by Charles E. Wyman.

Charles E. Wyman (1996). Handbook on Bioethanol: Production and Utilization. Applied Energy Technology Series, 424 pages.

D. Martinez, J. et al. (2009). Genome, transcriptome, and secretome analysis of wood decay fungus Postia placenta supports unique mechanisms of lignocellulose conversion. Proceedings of the National Academy of Sciences of the United States of America, 106(6), 1954-9.

Donald Klass (1998). Biomass for renewable energy, fuels, and chemicals. Academic Press. Ebook ISBN: 9780124109506. Published date: 25 th June 1998, USA.

D. Yogi Goswami, Frank Kreith (2016). Energy efficiency and renewable energy handbook, Boca Raton, London, New York.

Fan Cong (2011). A strategy to facilitate the conversion of lignocellulose to bioethanol via lignin modification. The Pennsylvania State University.

Galbe M. and Zacchi G. (2002). A review of the production of ethanol from softwood. Appl Microbiol Biotechnol, 59(6), 618-28. Epub 2002 Jul 17.

Hartwell LH, Unger MW (1977). Unequal division in Saccharomyces cerevisiae and its implications for the control of cell division. J Cell Biol, 75, 422-435.

Ho NW, Chen Z, Brainard AP (1998). Genetically engineered Saccharomyces yeast capable of effective cofermentation of glucose and xylose. Appl Environ Microbiol, 64(5),1852-1859.

Ingledew WM (1999). Alcohol production by Saccharomyces cerevisiae: a yeast primer. In The alcohol textbook, 3rd, Nottingham University Press, UK.

Javis M (2003). Chemistry: Cellulose stacks up. Nature 426:611-612

Glasser WG (1980). Lignin. In: Casey JP, ed. Pulp and paper: Chemistry and chemical technology, Vol. 3, ${ }^{\text {rd }}$ ed. Wiley-Interscience, New York, pp. $39-11$.

Kumar P, Barrett DM, Delwiche MJ, Stroeve P. (2009). Methods for pretreatment of lignocellulosic biomass for efficient hydrolysis and biofuel production. Ind Eng Chem, 48(8), 3713-29.

Kim T.H. (2013). Biomass - from fundamentals to applications. Kongju National University publishing, 242 pages.

Kim Olofsson, Magnus Bertilsson and Gunnar Liden (2008). A short review on SSF - an interesting process option for ethanol production from lignocellulosic feedstocks. Biotechnology for Biofuels, 1:7 dol: 10.1 186/1754-6834-1-7.

Mohammad J. Taherzadeh and Keikhosro Karimi (2007). Enzymatic-based hydrolysis processes for ethanol. 2(4). Access in http://ncsu.edu/bioresources ISSN: 1930-2126

Naik, S.N.; Goud, V.V.; Rout, P.K.; Dalai, A.K. (2009). Production of first and second generation biofuels: A comprehensive review. Renew. Sustain. Energy Rev, 14, 578-597.

Patnaik PR (2003). Oscillatory metabolism of Saccharomyces cerevisiae: an overview of mechanisms and models. Biotechnol. Adv., 21, 183-192.

P.F.H. Harmsen, W.J.J Huijgen, L.M. Bermudez Lopez, R.R.C. Bakker (2010). Literature review of physical and chemical pretreatment processes for lignocellulosic biomass. 
Wageningenur University \& Research centre - Food \& Biobase research, Netherlands.

Sakanen KV, Ludwig CH, eds. (1971). Lignin: Occurrence, formation, structure and reactions. Wiley-Interscience, New York.

Sluiter, A., B. Hames, R. Ruiz, C. Scarlata, J. Sluiter, D. Templeton, and Crocker, D. (2008). Determination of Structural Carbohydrates and Lignin in Biomass. NREL Laboratory Analytical Procedures for standard biomass analysis.

Sjostrom E (1993). Wood chemistry: Fundamanetals and applications, $2^{\text {nd }}$ ed. Academic Press, London, UK.

S.N. Naik, Vaibhav V. Goud, Prasant K. Rout, Ajay K. Dalai (2009). Production of first and second generation biofuels: A comprehensive review. Renewable and sustainable energy review 14 (2010) 578-597.

Yan Lin, Shuzo Tanaka (2005). Ethanol fermentation from biomass resources: current state and prospects. Appl Microbiol Biotechnol (2006) 69: 627-642 DOI 10.1007/s00253-005-0229. 